\title{
Monolithically integrated DFB lasers for tunable and narrow linewidth mm-wave generation
}

\author{
Marco Zanola, Michael J. Strain, Member, IEEE, Guido Giuliani, Member, IEEE, \\ and Marc Sorel, Member, IEEE
}

\begin{abstract}
A monolithic optoelectronic device for the generation of tunable and narrow linewidth millimeter-wave signals is presented. The device consists of three mutually injected DFB lasers whose emission frequencies are stabilized via a Four Wave Mixing process. Their beating on high-speed photodetector generates a narrow linewidth electrical signal that is continuously tunable over several tens of GHz.
\end{abstract}

Index Terms - mm-waves, integrated optics, DFB lasers, Photomixing

\section{INTRODUCTION}

$\mathrm{I}_{1}$ $\mathrm{n}$ the last few years the interest in mm-waves and $\mathrm{THz}$ technologies has steadily increased, since the availability of sources and detectors capable of emitting efficiently in this frequency range [1-4] has the potential to impact on wide range of applications, such as spectroscopy, tomography, gas sensing and security. The technologies available to date for the generation of $\mathrm{CW} \mathrm{mm}$-wave and $\mathrm{THz}$ signals span from electronics [5] to photonics [6]. Traditional electronic approaches include impact avalanche transit time (IMPATT) diodes, Gunn diodes and frequency multipliers, and are capable of achieving $\approx 1 \mathrm{~Hz}$ linewidth, at the expense of a bulky set-up and poor tunability [7]. More recent microelectronic circuit technologies have enabled the demonstration of oscillators at hundreds of $\mathrm{GHz}$ with good power efficiency, but still with a very limited tunability $[8,9]$.

Manuscript received Oct XXX, XX; revised XXX, XX; accepted April $\mathrm{XX}, \mathrm{XX}$. Date of publication XX, XX; date of current version XX XX, XX .

M. Zanola is with the School of Engineering, University of Glasgow, Glasgow G12 8LT, U.K., on leave from the Dipartimento di Ingegneria Industriale e dell'Informazione, Università di Pavia, Pavia, Italy (e-mail: Marco.Zanola@glasgow.ac.uk).

M. J. Strain and M. Sorel are with the School of Engineering, University of Glasgow, Glasgow G12 8LT, U.K. (e-mail: Michael.Strain@glasgow.ac.uk; Marc.Sorel@glasgow.ac.uk).

G. Giuliani is with the Dipartimento di Ingegneria Industriale e dell'Informazione, Università di Pavia,Pavia 27100, Italy (e-mail: guido.giuliani@unipv.it).

Digital Object Identifier XZXXX/XXXX.
Photonic technologies have experienced the rapid growth of $\mathrm{THz}$ Quantum Cascade Lasers, that now offer $\mathrm{CW}$ emission at moderate powers with some tunability, while still requiring bulky external systems for cooling down to cryogenic temperatures [10]. The only approach that guarantees a tunability over more than a frequency decade is photomixing $[11,12,13]$, which is based on the coherent superposition of two single mode lasers, emitting at the frequencies $v_{1}$ and $v_{2}$, onto an ultrafast non-linear material that down-converts the optical signals and generates a RF electrical signal at the frequency difference $v_{\mathrm{RF}}=\left|v_{1}-v_{2}\right|$. This approach can be readily integrated on chip [14], and allows for easy tunability through the tuning of the optical frequency emitted by one or both lasers. However, in its standard configuration the photomixing technique offers a limited spectral purity of the generated signal, because the lasers are operating in a freerunning regime and their instantaneous frequency fluctuations are uncorrelated. Thus, the linewidth of the down-converted electrical signal is at best twice as large as the optical linewidth of the two lasers but can be much wider in the presence of uncorrelated thermal drifts of the two optical sources. A very successful solution for improving the stability is based on the phase locking of the two optical sources via an optical phase-lock loop. The performance in terms of phase noise of this approach is still unrivalled but the requirement for a reference microwave source limits the maximum frequency to a few hundreds of $\mathrm{GHz}$ and increases the complexity and cost of the source [15]. Enhanced performance of RF signal linewidths have also been demonstrated by way of dual laser master-slave injection locking schemes[16], though these
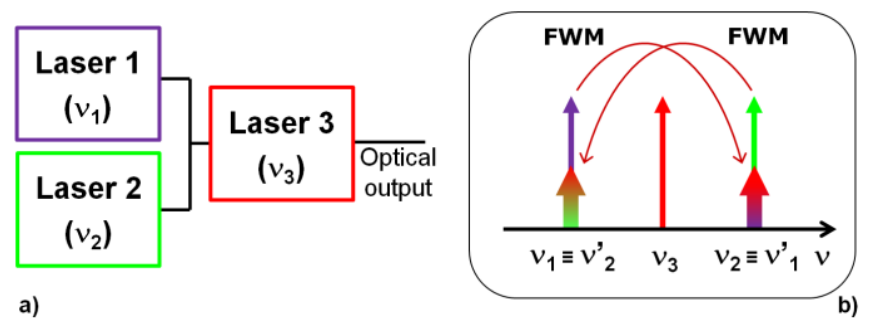

a)

Fig. 1. (a) Laser configuration for the Photomixing assisted by mutual injection locking and FWM technique (b) Mutual injection locking condition 
require the use of optical isolators that make them unsuitable for integration on a single chip.

A novel and compact scheme that has the potential to substantially improve the spectral purity of photomixing signals is based on the photomixing assisted by mutual injection locking via Four Wave Mixing (FWM) [17]. In this scheme, the frequency stability of the RF signal is enhanced by introducing a feedback effect on the instantaneous emission frequencies of the two semiconductor lasers that generate the beating signal. This can be obtained by adding a third laser, operating at the frequency $v_{3}$, as depicted in Fig.1a. Laser 1 and laser 2 are injected into laser 3, where FWM generates two new conjugate FWM signals that are "copies" of the lasers 1 and 2 at frequencies $v_{1}^{\prime}=2 v_{3}-v_{1}$ and $v_{2}^{\prime}=2 v_{3}-v_{2}$, respectively. When laser 3 is operating at the frequency $v_{3}=$ $\left(v_{2}+v_{1}\right) / 2$ the conjugate FWM signals have frequencies $v_{1}^{\prime}=$ $v_{2}$ and $v_{2}^{\prime}=v_{1}$. In this condition, laser 1 and laser 2 are mutually coupled via their conjugate FWM signals, and this causes a locking of these two laser oscillators (Fig. 1b). The frequency difference between all the three lasers is rigidly locked, and the instantaneous frequency fluctuations of the lasers are strongly correlated, with the positive consequence that the beating of laser 1 and laser 2 now generates a RF signal with a linewidth that is much narrower than that of the free-running lasers. The proposed scheme represents an evolution of the experiment by Goto et al. [18], where the mutual injection locking was achieved via FWM between two adjacent longitudinal modes of a Fabry-Perot laser.

In this paper we present a semiconductor monolithic photonic device for the generation of tunable and narrow linewidth $\mathrm{mm}$ wave signals, based on the novel scheme of photomixing assisted by mutual injection locking via Four Wave Mixing. Section II reports on the design and fabrication of the integrated monolithic device; Section III presents the experimental characterisation of the individual single mode lasers; finally, Section IV reports on the experimental results of mutual injection locking and RF signal generation.

\section{DEVICE DESIGN AND FABRICATION}

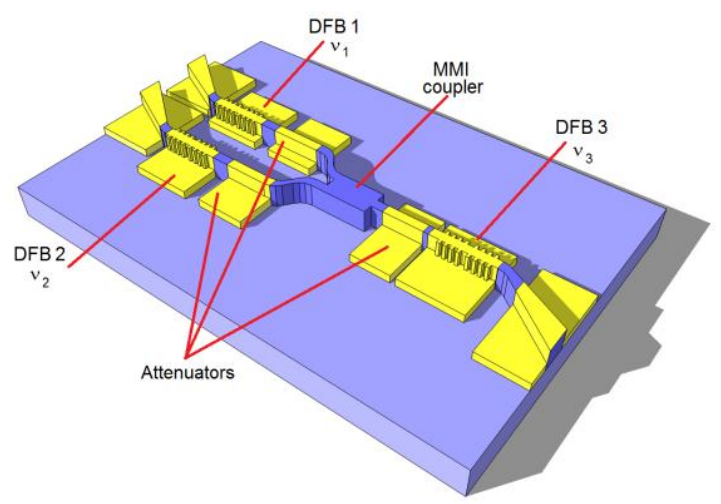

Fig. 2. Schematic of the monolithic device.

The mutual injection locking scheme, outlined above, relies on the generation of optical clones of two signals around a common pump and the re-injection of these new signals back into the original optical sources. Therefore, an integrated device implementation of this scheme requires three fundamental elements: (i) Three single mode DFB lasers operating at the frequencies $v_{1}, v_{2}$, and $v_{3}$; (ii) a non-linear section where the Four Wave Mixing conjugate signals can be generated; (iii) a feedback mechanism to allow the re-injection

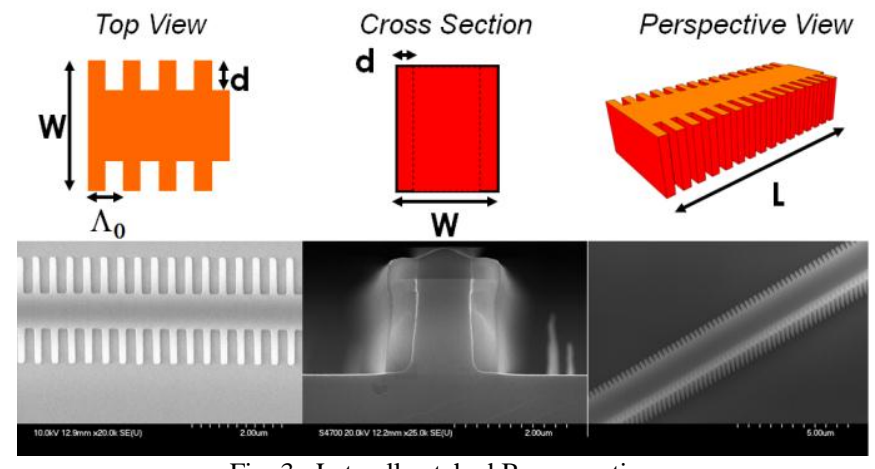

Fig. 3. Laterally etched Bragg gratings

of the newly generated signals into the original lasers. Fig. 2 shows a schematic of the integrated device developed in this work. DFB 1 (operating at $\left.v_{1}\right)$ and DFB $2\left(v_{2}\right)$ were coupled into DFB $3\left(v_{3}\right)$, where, due to the high non-linearity of the active material, Four Wave Mixing conjugate signals at the idler frequencies $v_{1}^{\prime}$ and $v_{2}^{\prime}$ were generated. The grating of DFB 3 also provided the feedback mechanism necessary for the mutual locking, by back-reflecting the newly generated signals towards the original lasers. The mutual coupling of the three lasers was achieved by a 2x1 MMI coupler [19], while attenuators were used in order to tune the levels of mutually injected optical power. The output waveguides were tilted at $10^{\circ}$ and up-tapered in order to avoid back-reflections from the cleaved facets. In order to employ the post-growth fabrication process already described in [20], gratings were obtained by a lateral etch of the waveguides (Fig.3). Each grating was $400 \mu \mathrm{m}$ long, the lateral recess $d$ was $300 \mathrm{~nm}$ and the waveguide width $W$ was $2.4 \mu \mathrm{m}$, allowing for a coupling coefficient $\kappa$ of $40 \mathrm{~cm}^{-1}$. A $\lambda / 4$ phase-shift section placed in the middle of the grating ensured the single mode operation of the lasers [21].

The device was fabricated on AlGaInAs/InP material, the etch mask was patterned by electron beam lithography into negative tone Hydrogen SilsesQuioxane (HSQ) resist and the waveguides were defined by RIE using a chemistry of $\mathrm{CH}_{4} \backslash \mathrm{H}_{2} \backslash \mathrm{O}_{2}$. The electrical isolation layer was realized deposing PECVD $\mathrm{SiO}_{2}$ silica and $\mathrm{HSQ}$, finally followed by the deposition of the p-type and n-type contacts.

\section{DEVICE CHARACTERISATION}

The lasing characteristics of the single DFB lasers were first evaluated individually. All three of the lasers exhibited threshold currents of $25 \mathrm{~mA}$ and output powers in air up to $10 \mathrm{~mW}$, operating in single mode regime with a Side Mode Suppression Ratio (SMSR) exceeding 60 dB (Fig.4a). 
Fig. $4 \mathrm{~b}$ shows the emitted wavelength as a function of the DFB 3 bias current: a continuous wavelength tuning of $3.5 \mathrm{~nm}$ is achievable by tuning the injected current from threshold up to $150 \mathrm{~mA}$, giving a wavelength tuning of $\sim 24 \mathrm{pm} / \mathrm{mA}$, or $\sim 3 \mathrm{GHz} / \mathrm{mA}$.

a)

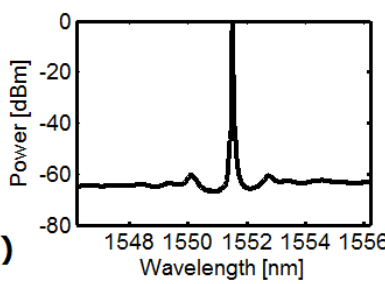

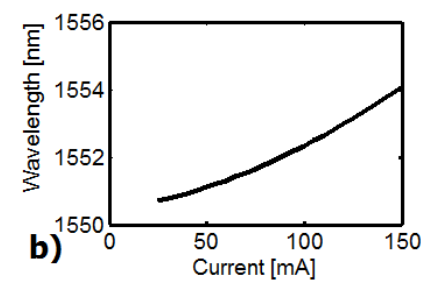

Fig. 4. (a) Optical spectrum of a single DFB laser; (b) emission wavelength as a function of the injected current.

The optical linewidth $\Delta v_{\text {opt }}$ of each DFB laser was measured using a coherent heterodyne technique, and the linewidth was calculated using a Lorentzian fitting. All of the lasers showed an optical linewidth $\Delta v_{\text {opt }}$ of $13 \mathrm{MHz}$.

The intra-cavity FWM conversion efficiency of the DFB lasers was characterised on a purposely developed integrated device that consisted of two lasers in a back-to-back configuration separated by an attenuator (see inset of Fig.5b.) The output facets of both lasers were cleaved at an angle of 10 degrees and the design parameters of the DFB lasers were identical to those described in section II. DFB 1 was biased at a constant current of $100 \mathrm{~mA}$, whereas the current of DFB 2 was swept from $100 \mathrm{~mA}$ to $230 \mathrm{~mA}$ in order to tune the frequency difference between the lasers from $0 \mathrm{GHz}$ to 400 $\mathrm{GHz}$. The attenuator between the lasers was adjusted to ensure a constant power injection from DFB 2 to DFB 1 of
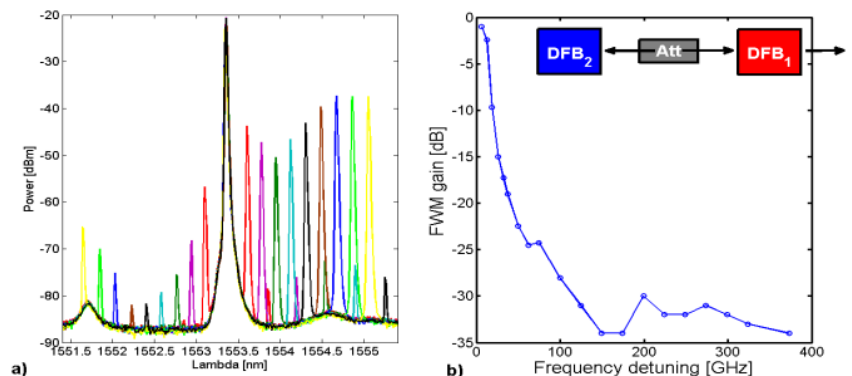

Fig. 5. (a) Optical spectrum and (b) FWM gain as a function of the detuning between the injected signals

approximately $-20 \mathrm{dBm}$ for all values of frequency detuning. The optical spectra collected from the output facet of DFB 1 for different frequency detunings are shown in Fig.5a. Both the injected mode from DFB 2 and the conjugated FWM mode generated by the interaction of DFB 1and DFB 2, located at the frequency $v_{2}^{\prime}=2 v_{1}-v_{2}$, are clearly visible.

Fig. 5b shows the FWM gain as a function of the frequency detuning, where $\mathrm{FWM}_{\text {gain }}=\mathrm{P}\left(v_{2}^{\prime}\right) / \mathrm{P}\left(v_{2}\right), \mathrm{P}\left(v_{2}\right)$ being the power injected into DFB 1 by DFB 2, and $\mathrm{P}\left(v_{2}^{\prime}\right)$ the power of the conjugate FWM beam. As expected, the rich variety of dynamical processes in semiconductor gain media leads to a FWM spectrum that exhibits pronounced frequency dependence. Non-linearities originated by band-to-band transitions are dominant for low frequency detunings and rapidly decrease for detunings larger than the inverse of the carrier lifetime [22]. In the presence of a DFB laser, this frequency dependence of the FWM is multiplied by the transfer function of the grating [23], which generates a strong enhancement of the FWM gain. It is worth pointing out that cavity-enhanced FWM occurs over a frequency detuning that can be much larger than the grating stopband because of the presence of the grating side lobes. In our devices, an enhanced FWM gain occurs at frequencies corresponding to the side lobe peaks at $200 \mathrm{GHz}$ and $280 \mathrm{GHz}$, as shown in Fig. 5 b.

\section{MUTUAL INJECTION-LOCKING EXPERIMENTS}

\section{A. Two DFB locking}

The locking of two mutually injected lasers operating at the same frequency was investigated in order to assess the levels of optical injection necessary to lock these lasers and the
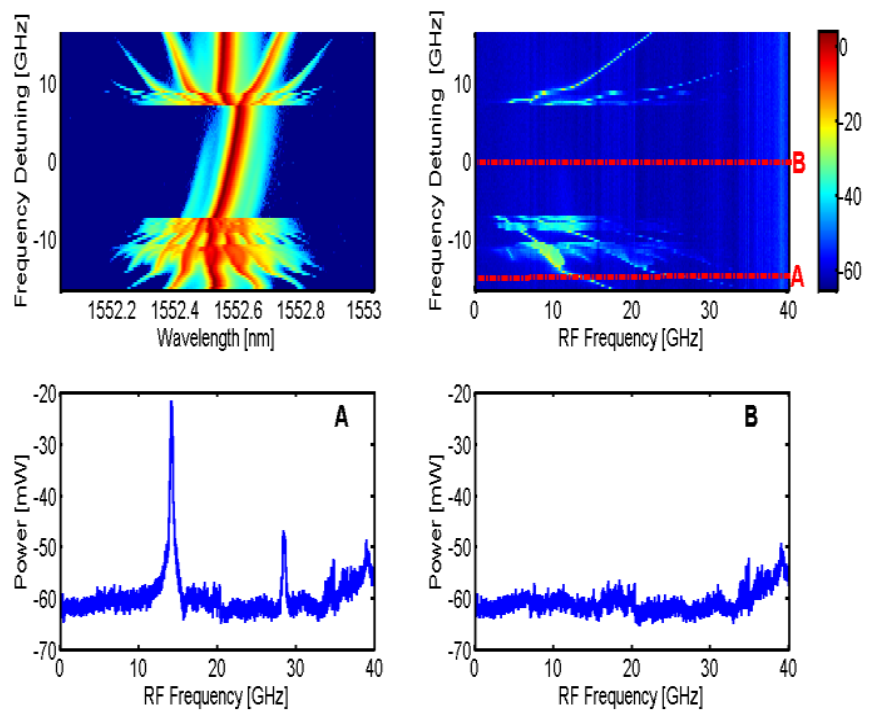

Fig. 6. Evolution of the optical (top left) and electrical (top right) spectra during the tuning of current of DFB $1\left(\mathrm{I}_{\mathrm{DFB}_{-} 1}\right)$. The cross-sections A and B show the electrical spectrum for different DFB 1 currents.

dependence of the locking range on the mutually injected power. The locking range can be defined as the interval during which the two lasers are phase correlated and operating at the same frequency as a function of the detuning between their free running wavelengths. The experiments were carried out using the two-laser device already described in Section III. DFB 2 was biased at a constant current of $100 \mathrm{~mA}$, while the current of DFB 1 was swept from $94 \mathrm{~mA}$ to $106 \mathrm{~mA}$ in order to tune its operating wavelength across that of DFB 2 . Different values of the injection levels were obtained by reverse biasing the attenuator between DFB 1 and DFB 2. The signal was collected by a lensed optical fiber and sent to an Optical Spectrum Analyser (OSA) and to a high speed photodiode; an Electrical Spectrum Analyser (ESA) was used to record the spectrum of the generated beating signals. Figure 6 shows the evolution of the optical (top left) and electrical (top right) spectra during the tuning of current of DFB 1 ( $\mathrm{I}_{\mathrm{DFB}}$ 1). For a free running frequency detuning greater than $\pm 7 \mathrm{GHz}$ the two lasers were unlocked, generating a broad linewidth electrical signal (cross-section A). For a frequency detuning lower than $\pm 7 \mathrm{GHz}$ the two lasers were mutually phase-locked and emitted at identical optical frequencies. In the electrical 


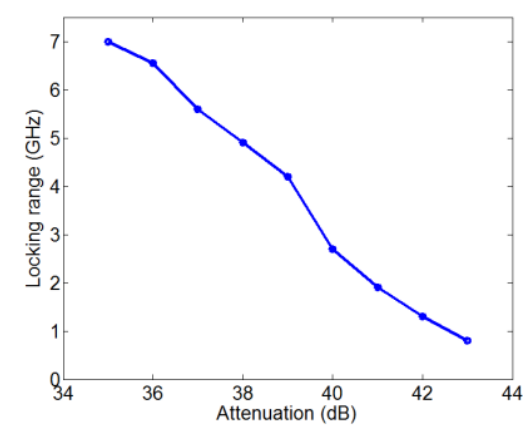

Fig. 7. Locking range as a function of the attenuation between the two lasers

spectrum this corresponds to the absence of any beating signals other that the $\mathrm{CW}$ term (cross-section $\mathrm{B}$ ). The experiment was then repeated for different values of attenuation between the lasers. Figure 7 shows the locking range (measured as double-sided free-running frequencies detuning) as a function of the attenuation between the lasers. As expected the locking range decreases for increasing optical attenuation between the lasers. For attenuations smaller than $35 \mathrm{~dB}$ an unstable locking regime was achieved, which was characterized by unstable dynamics of the laser emission wavelengths and beating signals. For attenuations between 35 $\mathrm{dB}$ and $42 \mathrm{~dB}$ a stable locking regime was achieved. Finally, for attenuation larger than $43 \mathrm{~dB}$, mutual injection locking could not be achieved, because the mutual coupling was too weak.

As a confirmation of the mutual locking, the optical linewidths of both DFB 1 and DFB 2 were measured in unlocked and locked conditions. Their optical linewidths were $13.3 \mathrm{MHz}$ in the unlocked case whereas, when the lasers were locked, their linewidths reduced to $6 \mathrm{MHz}$, as similarly reported in [24].

\section{B. Three DFB locking}

As described in Section I, the locking condition for the threelaser system requires the DFB 3 to lase at the frequency $v_{3}=$
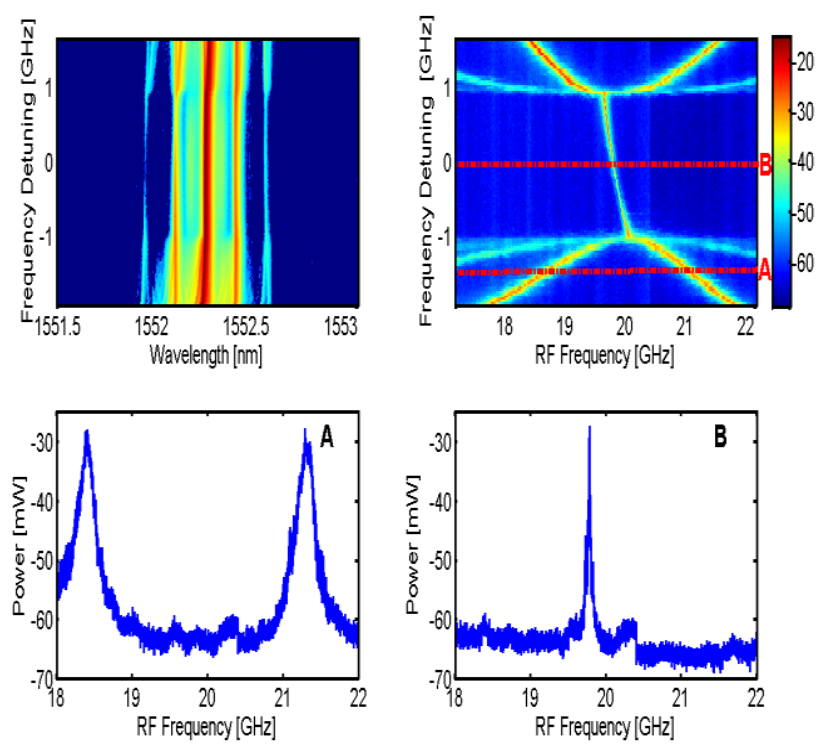

Fig. 8. Evolution of the optical (top left) and electrical (top right) spectra

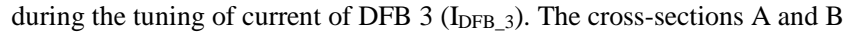
show the electrical spectrum for different DFB 3 currents. $\left(v_{2}+v_{1}\right) / 2$ between DFB 1 and DFB 2. The locking of the three lasers was verified by biasing them close to the locking condition and then fine tuning the frequency of DFB 3 by current tuning. The optical output of the device was beat on a fast photodiode (50GHz bandwidth) in order to generate the RF electrical signal. Fig. 8a,b show the optical and electrical RF signal spectra as a function of DFB 3 detuning across $\left(v_{2}+\right.$ $\left.v_{1}\right) / 2$, while cross-sections $\mathrm{A}$ and $\mathrm{B}$ show the detail of the electrical spectra at $0 \mathrm{GHz}$ and $1.5 \mathrm{GHz}$ detuning, respectively. For a DFB 3 detuning greater than $\pm 1 \mathrm{GHz}$, the three laser system did not lock. The broad RF peaks in cross-section (A) represent the beating between the laser couples (DFB 1 and DFB 3) and (DFB 3 and DFB 2). The RF linewidths of the beat signals in this case were $27 \mathrm{MHz}$, calculated from a Lorentzian fit of the measured signals. For a frequency detuning of DFB 3 of less than $\pm 1 \mathrm{GHz}$ from $\left(v_{2}+v_{1}\right) / 2$, the three laser system clearly show stable locking. This condition is characterized by the generation of a single RF beating signal with a linewidth of $2.5 \mathrm{MHz}$ as illustrated in cross-section (B). An abrupt decrease in the RF linewidth is observed at the edge of the locking region as shown in Fig.9. In the unlocked condition (Fig.9, red line) $\Delta v_{R F}$ was $27 \mathrm{MHz}$, while in locked conditions (Fig.9a, blue line) $\Delta v_{\mathrm{RF}}$ decreased by more than one order of magnitude to $2.5 \mathrm{MHz}$.

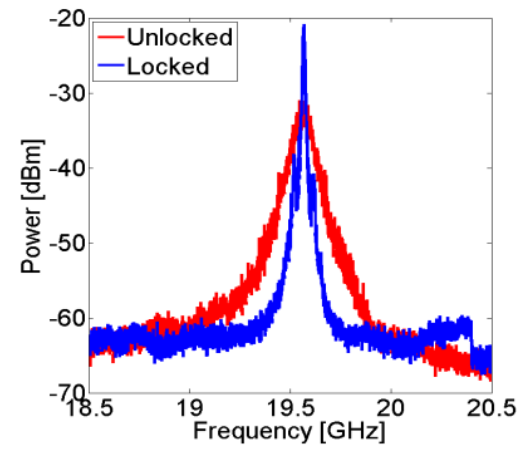

Fig. 9. RF signal in unlocked and locked conditions $\underline{(\mathrm{RBW}: 500 \mathrm{kHz})}$

As previously discussed for the mutual injection of two DFBs, the locking regime was also verified by measuring the optical linewidths $\Delta v_{\mathrm{opt}}$ of the three lasers during the locking process. When unlocked, the lasers showed optical linewidths $\Delta v_{\text {opt }}$ of $13 \mathrm{MHz}$, whereas in locked conditions $\Delta v_{\text {opt }}$ decreased to $6 \mathrm{MHz}$ for all of the three lasers. Therefore, the observation of a RF beating linewidth below the optical linewidth of each of the individual lasers represents the experimental signature of the phase-locking between all three lasers.

As an improvement of the Photomixing technique, this new method is expected to preserve the wide and continuous tunability of the generated RF signal. By simply tuning the biasing current of the three lasers it was possible to change their emission frequencies, and therefore continuously tune the frequency of the generated RF signal, as shown in Fig. 10a. In order to access this continuous tuning range the three DFBs must be precisely spaced in frequency by fabrication[20] otherwise differences in injection currents required to set the laser wavelengths will result in large power output discrepancies and therefore prevent locking. Fig. 10b shows 
the tuning of the RF signal over a wide range of frequencies. The low-frequency limit of tunability is limited to $5 \mathrm{GHz}$ by the occurrence of frequency locking of all the three lasers, that collapse into a single emission frequency (strong mutual coupling), while the upper limit is set by the efficiency of the FWM process used to stabilize the three lasers (weak mutual coupling).
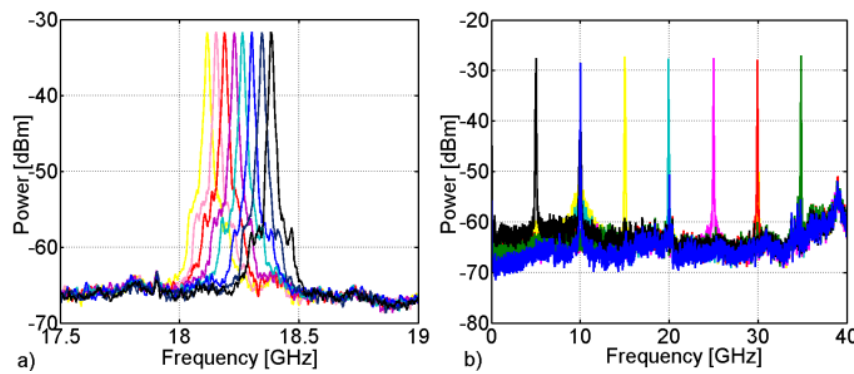

Fig. 10. (a) Fine and (b) coarse tunability of the RF beating signal

The RF beat signal linewidth was measured over the full range of generated signals from $5 \mathrm{GHz}$ to $40 \mathrm{GHz}$, in locked condition. The average linewidth was $2.5 \mathrm{MHz}$, with a standard deviation of $0.3 \mathrm{MHz}$ across the full range demonstrating the flexibility of this generation technique over a wide range of signal frequencies.

\section{CONCLUSIONS}

We have presented a monolithic optoelectronic device for the generation of tunable and narrow-linewidth mm-wave generation based on the newly proposed Photomixing assisted by mutual injection locking and Four Wave Mixing technique.

A mutual injection mechanism was used to stabilise the emission frequencies of three DFB lasers. Their beating on an external photodetector generated a RF signal with a linewidth of $2.5 \mathrm{MHz}$. The frequency tunability of the system as well as the maximum mm-wave frequency that can be achieved is defined by the bandwith over which FWM conjugate signals are generated and fed back into the DFB lasers. In the devices we presented here, the maximum frequency is limited by the stopband of the DFB gratings to approximately $350 \mathrm{GHz}$. Because the bandwidth of the FWM can easily exceeds several $\mathrm{THz}$ in DFB lasers [23], a much wider tunability of the operating frequency can be simply obtained by using gratings with larger stopbands.

\section{ACKNOWLEDGEMENTS}

The authors would like to thank the staff of the James Watt Nanofabrication Centre. This work was supported by Fondazione CARIPLO (grant\# 2007-5263), and EPRSC (EP/E065112/1).

\section{REFERENCES}

[1] Hongqian Sun; Ding, Y.J.; Zotova, I.B.; , "THz Spectroscopy by Frequency-Tuning Monochromatic THz Source: From Single Species to Gas Mixtures," Sensors Journal, IEEE , vol.10, no.3, Mar. 2010

[2] S. Wang, B. Ferguson, D. Abbott, and X.-C. Zhang, "T-ray imaging and tomography," J. Biol. Phys., vol. 29, no. 2-3, pp. 247-256, 2003
[3] D. Mittleman, R. Jacobsen, R. Neelamani, R. Baraniuk, and M. Nuss, "Gas sensing using terahertz time-domain spectroscopy," Applied Physics B: Lasers and Optics, vol. 67, pp. 379-390, 1998.

[4] C. Baker, T. Lo, W. R. Tribe, B. E. Cole, M. R. Hogbin, and M. C. Kemp, "Detection of concealed explosives at a distance using $\mathrm{THz}$ technology," Proc. IEEE, vol. 95, no. 8, pp. 1559-1565, Aug. 2007.

[5] Jae-Sung Rieh; Sanggeun Jeon; Moonil Kim; , "An overview of integrated $\mathrm{THz}$ electronics for communication applications," Circuits and Systems (MWSCAS), 2011 IEEE 54th International Midwest Symposium on , vol., no., pp.1-4, 7-10 Aug. 2011

[6] Saeedkia, D.; Safavi-Naeini, S.; , "Terahertz Photonics: Optoelectronic Techniques for Generation and Detection of Terahertz Waves," Lightwave Technology, Journal of , vol.26, no.15, Aug.1, 2008

[7] S. Schiller, B. Roth, F. Lewen, O. Ricken, M.C. Wiedner, "Ultranarrow-linewidth continuous-wave $\mathrm{THz}$ sources based on multiplier chains", Appl. Phys. B, vol. 95, pp. 55-61, 2009.

[8] E. Seok, C. Cao, D. Shim', D.J. Arenas', D.B. Tanner', C.-M, Hung, K. O. Kenneth, "A 410GHz CMOS Push-Push Oscillator with an On-Chip Patch Antenna", Proceedings of IEEE International Solid-State Circuits Conference - ISSCC, pp. 472-473, 2008.

[9] O. Momeni, E. Afshari, "High power terahertz and millimeter-wave oscillator design: a systematic approach", IEEE J. Solid-State Circ., , vol. 46, no. 3 pp. 583-597, 2011.

[10] S. Kumar, "Recent progress in Terahertz Quantum Cascade Lasers", IEEE J. Sel. Topics Quantum Electron., vol. 17, no. 1, pp. 38-47, 2011

[11] A.J. Seeds, "Microwave photonics", IEEE Transactions on Microwave Theory and Techniques, vol. 50, no. 3, pp. 877-887, 2002

[12] A. Stöhr, D. Jäger, "Optical generation of mm-wave signals", in: Microwave Photonics, S. Iezekiel, Ed., John Wiley \& Sons, 2008

[13] B. Sartorius, D. Stanze, T. Göbel, D. Schmidt, M. Schell, "Continuous Wave Terahertz Systems Based on $1.5 \mu \mathrm{m}$ Telecom Technologies", J Infrared Milli Terahz Waves, vol. 33, pp. 405-417, 2011.

[14] N. Kim, S. Han, H. Ryu, H. Ko, J. Park, D. Lee, M. Jeon, and K. Park, "Distributed feedback laser diode integrated with distributed Bragg reflector for continuous-wave terahertz generation," Opt. Express 20, $17496-17502,2012$

[15] R. J. Steed, L. Ponnampalam, M. J. Fice, C. C. Renaud, D. C. Rogers, D. G. Moodie, G. D. Maxwell, I. F. Lealman, M. J. Robertson, L. Pavlovic, L. Naglic, M. Vidmar, and A. J. Seeds, "Hybrid integrated optical phase-lock loops for photonic terahertz sources," J. Sel. Top. Quantum Electron. 17(1), 210-217 (2011).

[16] Y.-S. Juan and F.-Y. Lin, "Photonic Generation of Broadly Tunable Microwave Signals Utilizing a Dual-Beam Optically Injected Semiconductor Laser," IEEE Photonics Journal, vol.3, no. 4, Aug. 2011.

[17] Soldo, M.; Gibbons, N.; Giuliani, G.; "Generation of a narrow linewidth mm-wave signal from two phase-locked DFB lasers that are mutually coupled via four wave mixing,". CLEO Europe - EQEC 2009, 14-19 June 2009

[18] Goto, R.; Goto, T.; Kasuya, H.; Mori, M.; Yamane, K.; , "Mutual injection locking between two DFB LDs which lase at frequencies separated by one Fabry-Perot mode spacing," Electronics Letters , vol.34, no.17, pp.1669-1670, 20 Aug 1998

[19] Soldano, L.B.; Pennings, E.C.M.; , "Optical multi-mode interference devices based on self-imaging: principles and applications," Lightwave Technology, Journal of , vol.13, no.4, pp.615-627, Apr 1995

[20] Zanola, M.; Strain, M.J.; Giuliani, G.; Sorel, M.; , "Post-Growth Fabrication of Multiple Wavelength DFB Laser Arrays With Precise Wavelength Spacing," Photonics Technology Letters, IEEE , vol.24, no.12, pp.1063-1065, June15, 2012

[21] K. Utaka, S. Akiba, K. Sakai, and Y. Matsushima, \Analysis of quarterwave-shifted DFB laser," Electronics Letters, vol. 20, pp. 326, 1984.

[22] M. Yamada.: "Theoretical analysis of nonlinear optical phenomena taking into account the beating vibration of the electron density in semiconductor lasers', J. Appl. Phys., 66, pp. 81-89,1989.

[23] Minch, J.R.; Chih-Sheng Chang; Shun-Lien Chuang; , "Wavelength conversion in distributed-feedback lasers," Selected Topics in Quantum Electronics, IEEE Journal of , vol.3, no.2, pp.569-576, Apr 1997.

[24] S. Noda, K. Kojima, and K. Kyuma, "Mutual injection-locking properties of monolithically-integrated surface-emitting multiplequantum- well distributed feedback lasers," Quantum Electronics, IEEE Journal of, vol. 26, pp. 1883 \{1894, nov 1990. 
Marco Zanola graduated in Optoelectronic Engineering in 2005 and 2008 (bachelor and masters degrees) at the University of Pavia, with the thesis "Dynamic characterisation of Semiconductor Ring Lasers." He obtained his $\mathrm{PhD}$ in 2011, working on the generation of tunable and narrow linewidth $\mathrm{mm}$ wave signals using a monolithic optoelectronic device. During his research he moved to the University of Glasgow, Scotland, to design and fabricate the monolithic devices in the state-of-the-art cleanrooms of the James Watt Nanofabrication Centre. He is now working as Research Scientist at Sharp Laboratories of Europe.

Michael J. Strain received the MEng from the University of Strathclyde (UK), the M.A.Sc. from the University of Toronto (Canada), and the Ph.D. from the University of Glasgow (UK) in 2002, 2004, and 2008 respectively. In 2008 he joined the Department of Electronics and Electrical Engineering at the University of Glasgow as a post-doctoral researcher. His main research interests are in dispersion engineering, semiconductor mode-locked and ring laser geometries and silicon photonics.

Guido Giuliani is associate professor of optoelectronics at the Dipartimento di Ingegneria Industriale e dell'Informazione, University of Pavia, Pavia, Italy. His main research interests include semiconductor lasers, silicon photonics, RF signals and $\mathrm{THz}$ waves, optical sensors, laser interferometry, laser vibrometers and non-contact laser techniques for bio-medical measurements. Guido is co-founder of Julight Srl, a start-up Company that develops novel photonics solutions for sensors and instruments.

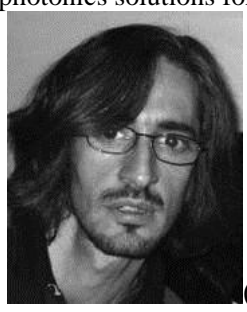

\section{(Guido Giuliani)}

Marc Sorel received the Electronics Engineering degree and the Ph.D. from the Universita di Pavia, Pavia, Italy, in 1995 and 1999, respectively. In 1998, he joined the Optoelectronics Group at the University of Glasgow, where he holds a senior lectureship position. His research interests include semiconductor technologies for monolithic and hybrid integration, optical sensors and ultrafast dynamics in semiconductor lasers. 